\title{
RECARGA GESTIONADA DE ACUÍFEROS: MULTIFUNCIONALIDAD EN LA ZONA REGABLE DE SANTIUSTE
}

\author{
Fernández Escalante, E. ${ }^{1}$, González Herrarte, F.B. ${ }^{2}$ y San Sebastián Sauto, J $(P)^{3}$ \\ ${ }^{1}$ Especialista en Planificación y Transferencia Tecnológica, I+D, Gerencia de Planificación Y \\ Transferencia, TRAGSA, efernan6@tragsa.es \\ 2 Técnico, Gerencia de Planificación y Transferencia, TRAGSA, fgonza13.ext@tragsa.es \\ 3 Jefe de Actuaciones y Proyectos, Gerencia de Ingeniería y Edificación, Tragsatec, Grupo \\ TRAGSA, Julián Camarillo 6, 28037 Madrid, jsss@tragsa.es
}

\section{Resumen}

Los dispositivos de recarga gestionada del acuífero de Santiuste (Segovia) dentro de la masa subterránea de Los Arenales llevan en funcionamiento desde 2002. Durante este tiempo se han realizado ampliaciones y modificaciones que han permitido pasar de un sistema puramente hidrogeológico para uso de riego a un dispositivo de gran flexibilidad y creciente multifuncionalidad. En este artículo se muestran las funciones que cumplen los diferentes dispositivos, incidiendo fundamentalmente en los factores ambientales que no aunque no formaban parte del planteamiento original han ganado protagonismo con la evolución del proyecto y su funcionamiento.

\section{Abstract}

Managed Aquifer Recharge (MAR) facilities of Santiuste within the groundwater body of Los Arenales (Segovia) have been in operation since 2002. During this time there have been additions and modifications that have allowed to move from a purely hydrogeologic scheme for irrigation purpose to a device of great flexibility and increased multifunctionality. This article shows the functions fulfilled by different devices, mainly focusing on the environmental factors that, even though they were not part of the original planning, have gained prominence with the evolution of the project and its operation.

\section{INTRODUCCIÓN: OBJETIVO DEL TRABAJO}

El acuífero de "Los Arenales" es un inmenso almacén arenoso de agua subterránea de más de 7.754 km² ubicado en Castilla y León. Desde mediados del siglo XX, la expansión del regadío condujo a una disminución del nivel de agua subterránea. La alarma saltó en 1992 cuando el Instituto Geológico y Minero de España (IGME) a través de su red de control constató el descenso de 30 metros en el nivel freático en Madrigal de las Altas Torres.

Por ello, el Ministerio de Agricultura impulsó la instalación de sistemas de recarga gestionada del acuífero en tres zonas piloto: Santiuste, Carracillo y Alcazarén. En 1992 se iniciaron los primeros estudios de viabilidad de recarga en el acuífero de los Arenales a través de la colaboración del Instituto Nacional de Reforma y Desarrollo Agrario (IRYDA) y el propio IGME. Con fecha 14 de Mayo de 1999, y una vez examinado el expediente incoado a instancias del Presidente de la Comunidad de Regantes, la Confederación Hidrográfica del Duero (CHD) resolvió otorgar la concesión de aprovechamiento de aguas superficiales a la CR de Regantes de la Cubeta de San Juan Bautista de $1.000 \mathrm{l} / \mathrm{s}$ del río Voltoya con toma en el término municipal de Aldeanueva del Codonal (Segovia) con destino a la recarga del acuífero de la cubeta de Santiuste. En 2000 se aprobó el proyecto original y en 2001 un modificado. Proyecto, obras, seguimiento y posterior vigilancia fueron confiados al Grupo Tragsa. 
Los primeros dispositivos se finalizaron un año más tarde, por lo que en 2012 se pudo celebrar una década de funcionamiento de la recarga en la cubeta de Santiuste. En este tiempo transcurrido, el sistema de recarga ha ido evolucionando desde una solución tecnológica con un enfoque inicial estrictamente hidrogeológico para un problema agronómico hasta un complejo sistema multifuncional donde se han ido incorporando nuevos criterios biológicos, químicos, socioeconómicos, administrativos, educativos...

La finalidad del presente trabajo es mostrar las múltiples capacidades de un sistema originalmente ideado para regadío y cómo valorar sus funciones y efectos técnicos, ambientales, económicos y sociales.

\section{DESCRIPCIÓN DE LOS DISPOSITIVOS DE RECARGA GESTIONADA}

Antes de pasar a definir los distintos dispositivos que constituyen el sistema de recarga es necesario dar unas pinceladas sobre las características hidrogeológicas del acuífero, aunque en la realidad el estudio en detalle de este apartado ocupó gran parte del esfuerzo técnico puesto que es fundamental para garantizar la viabilidad de la recarga.

\subsection{Descripción somera del acuífero de la Cubeta de Santiuste}

La Cubeta de Santiuste se extiende por $85 \mathrm{~km}^{2}$, limitado por los ríos Voltoya y Eresma por el Este y por afloramientos de baja permeabilidad del Terciario por el Oeste. Pese a su reducido tamaño, se considera un área de gran importancia para el regadío con aguas subterráneas. Los dispositivos de Recarga Gestionada de Acuíferos (MAR por su acrónimo en inglés) aprovechan el antiguo curso del arroyo de la Ermita (Figura 1), prácticamente desaparecido tras ser sometido a grandes movimientos de tierra durante las sequías de los últimos 30 años.

La traza del acuífero, según la geofísica (Galán, 2001), transcurre en una orientación de SSE a NNW hacia los dos ríos citados formándose 8 pequeñas cubetas interconectadas con base en el Terciario impermeable y separaciones formadas por afloramientos del mismo y a su vez cubiertas con depósitos de arena de origen eólico y fluvial de potencia variable pero gran trasmisividad (Figura 2). Uno de los afloramientos subdivide la zona en 2 mitades Norte y Sur.

\subsection{Descripción de los dispositivos de recarga}

Las obras de recarga en Santiuste se basan en 3 proyectos:

- Obra financiada por el Ministerio de Agricultura: "Proyecto de recarga del acuífero de la cubeta de Santiuste de San Juan Bautista (Segovia)" 2000-2002

- Obra financiada por la Consejería de Agricultura de la Junta de Castilla y León: "Obras de infraestructura en la zona de concentración parcelaria de la cubeta de Santiuste de San Juan Bautista (Segovia)" 2004-2006

- Obras de financiación local: "Acondicionamiento de infraestructuras en los ayuntamientos de Coca y Villeguillo" 2006-2008

La relación topológica e hidráulica entre los diferentes dispositivos descritos se muestran en el esquema de la Figura 3. 


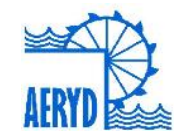

XXXIII Congreso Nacional de Riegos

Universitat Politècnica de València, Valencia 2015

DOI:http://dx.doi.org/10.4995/CNRiegos.2015.1445

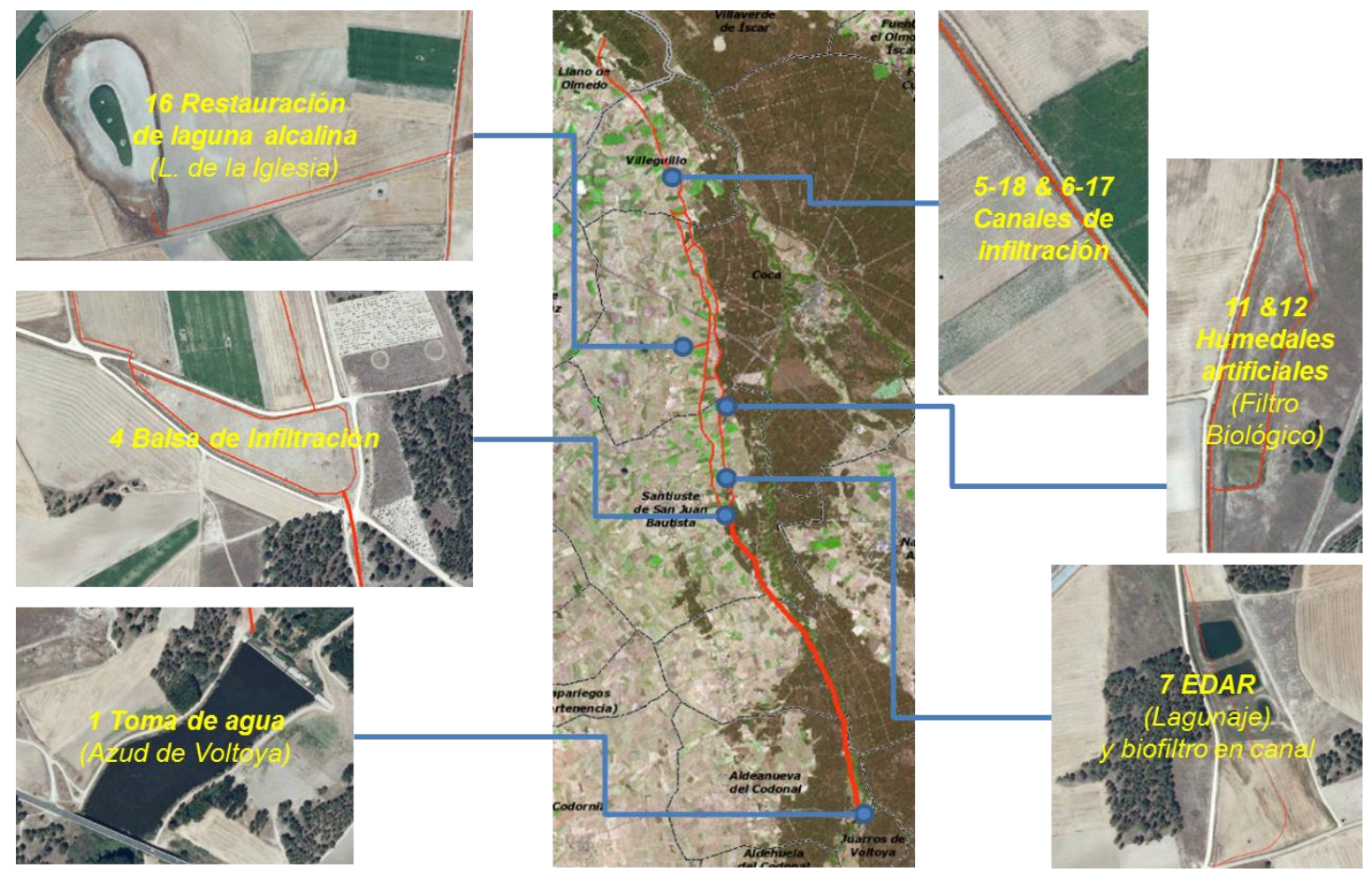

Figura 1. Vista en planta del sistema de recarga de Santiuste sobre ortofoto y principales dispositivos MAR
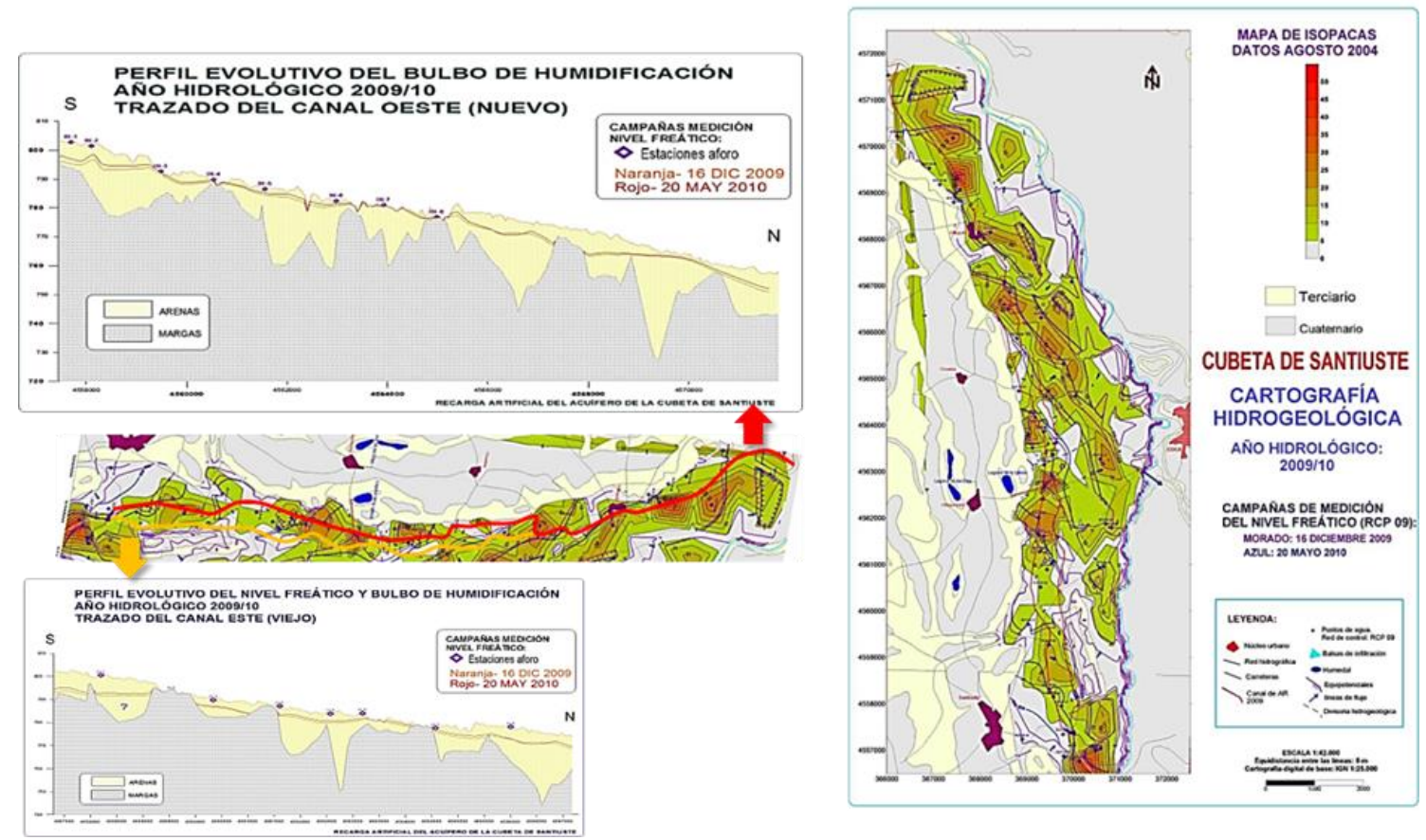

Figura 2. Vista en planta del sistema de recarga de Santiuste. Perfiles del canal Oeste (Caz Nuevo y extensión del Este) y Este (Caz Viejo)

\subsubsection{Azud del río Voltoya}

Azud de planta recta con perfil de vertedero tipo Creagel de $107 \mathrm{~m}$ de longitud, $3 \mathrm{~m}$ de altura y capacidad para $60.000 \mathrm{~m}^{3}$. La toma está diseñada para un caudal máximo de 1 $\mathrm{m}^{3} / \mathrm{s}$, siempre que se registre un caudal ecológico de la misma cuantía en el caudalímetro del Voltoya en Coca conectado en tiempo real a la Confederación Hidrográfica del Duero. La 
concesión dura de noviembre a mayo. La presa tiene un diseño específico con filtros, mecanismos de decantación y sistemas anti-entrada de peces.

\subsubsection{Tubería de conducción}

El agua del río Voltoya es desviada por flujo gravitacional de 816 a $812 \mathrm{~m}$ de cota a través de 9,82 km de tuberías enterradas a través del pinar de Pinus pinaster hasta el dispositivo de cabecera. La tubería es de poliéster reforzado con fibra de vidrio de $900 \mathrm{~mm}$ de diámetro y tiene 16 chimeneas de aireación y 10 desagües en su trayecto. La apertura es manual y la recarga no genera coste energético alguno al circular toda ella por gravedad.

\subsubsection{Balsa de decantación e infiltración}

La cabecera consta de una balsa de 1,4 ha tras un contador de flujo, un sistema de filtrado y algunos pozos de gran diámetro en el extremo sur del acuífero. Consta de un dispositivo de decantación de $36 \mathrm{~m}^{2}$ con disipador de energía para evitar erosión y burbujeo.

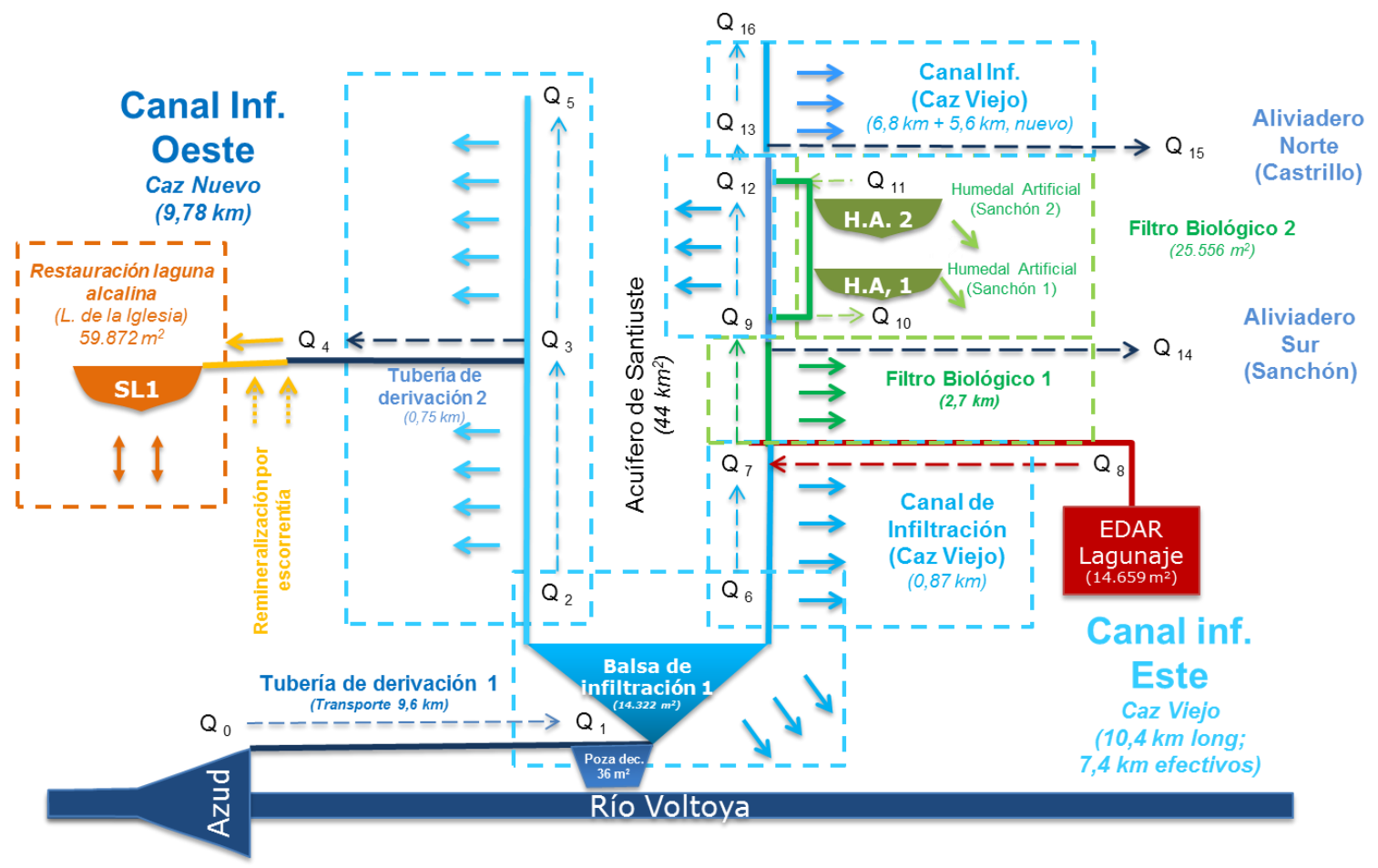

Figura 3. Esquema del sistema de recarga de Santiuste. Principales dispositivos y caudales de entrada y salida del sistema

\subsubsection{Canales de infiltración}

Dos "caces" en tierra $(1 * 1,5 \mathrm{~m}$, talud $1 / 1)$ circulan paralelos de sur a norte por el $20 \%$ del viejo curso del arroyo de la Ermita con una longitud de unos $25 \mathrm{~km}$ y un desnivel de 30 metros. Sirven como sistema principal de recarga y distribución siguiendo la localización de las 8 cubetas menores del acuífero. El caz viejo circula por el Este durante 10,7 km y el caz nuevo por el Oeste está operativo desde 2005-06 y ocupa $9,78 \mathrm{~km}$. Ambos cuentan con 5 interconexiones. El canal Este se extendió a partir de 2007-08 con 5,6 nuevos kilómetros más rodeando el núcleo de Villeguillo por el Este hasta el término de Llano de Olmedo. 


\subsubsection{Toma de derivación a Laguna de la Iglesia}

Una tubería de unos 750 m parte desde el caz Oeste hasta un dispositivo "en hongo" que descarga el agua hacia la laguna alcalina remineralizándose a medida que circula sobre los biosedimentos y costras salina de la playa perilagunar.

\subsubsection{Humedales Artificiales del Sanchón}

Los humedales de Sanchón son excavaciones realizadas por TRAGSA aprovechando el alto nivel piezométrico por ese lado del acuífero para formar 3 balsas denominadas Sanchón 1, 2a y 2b. La primera es un ensanchamiento del canal de $1.374 \mathrm{~m}^{2}$ fácilmente llenado por rebosamiento del canal. El segundo es una balsa cuadrangular de $2.418 \mathrm{~m}^{2}$ con orla de freatófilas y el tercero es una extensa balsa rectangular de $15.740 \mathrm{~m}^{2}$, cuya cota ligeramente superior a la continuación del canal Este hace más difícil su llenado.

\subsubsection{EDAR de lagunaje}

Se trata de una estación por lagunaje donde la capacidad de depuración es muy limitada según los datos de campo (Figura 5). Aporta anualmente 0,5 $\mathrm{hm}^{3}$ a la recarga desde 2005. Ocupa $14.659 \mathrm{~m}^{2}$ y da servicio al pueblo de Santiuste de San Juan Bautista. La EDAR, de los 17 compuestos considerados, aumenta de $200-400 \%$ el contenido en sulfatos, $\mathrm{Ca}, \mathrm{COD}, \mathrm{Cl}$, alcalinidad, dureza y As, de $400-500 \%$ la conductividad, $\mathrm{Mg}$ y $\mathrm{Na}$, de $500-$ $700 \%$ turbidez y nitratos, en casi $2.000 \% \mathrm{~K}$, P y fosfatos. La cafeína es un $5.600 \%$ y el amonio $14.211 \%$ mayor en el efluente. Sólo $\mathrm{Fe}$ y $\mathrm{Cu}$ son inferiores en el efluente de la depuradora (63 y $74 \%)$.

\section{RESULTADOS Y DISCUSIÓN}

Las diferentes funciones de los dispositivos son dependientes de su puesta en funcionamiento (Tabla 1. Cronología de dispositivos operativos en Santiuste 2002-2012 (F. Escalante, 2014)). A modo de ejemplo, la recarga por el canal Este ha sido constante desde el inicio del proyecto mientras que la de la Laguna de la Iglesia ha sido intermitente entre 2005 y 2011 con sólo 4 campañas de funcionamiento.

Tabla 1. Cronología de dispositivos operativos en Santiuste 2002-2012 (F. Escalante, 2014)

\begin{tabular}{|c|c|c|c|c|c|c|c|c|c|c|c|}
\hline DISPOSITIVOS & $\begin{array}{l}\text { FUNCIONES } \\
\text { PPALES. }\end{array}$ & 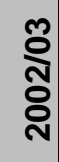 & $\begin{array}{l}\text { ঠ } \\
\text { 유 }\end{array}$ & 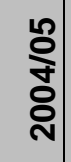 & 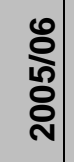 & $\begin{array}{l}\text { ㅇ. } \\
\text { ઠ̊. }\end{array}$ & $\begin{array}{l}\infty \\
\stackrel{0}{N} \\
\text { ర్సి }\end{array}$ & 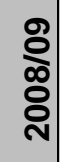 & $\begin{array}{l}0 \\
\text { 응 } \\
\text { 옹 }\end{array}$ & $\frac{5}{\frac{5}{0}}$ & $\frac{N}{\Sigma}$ \\
\hline Azud & Derivación & $x$ & $x$ & $x$ & $x$ & $x$ & $x$ & $x$ & $x$ & $x$ & \\
\hline Tubería & Transporte & $x$ & $x$ & $x$ & $x$ & $x$ & $\mathrm{x}$ & $x$ & $x$ & $x$ & \\
\hline Balsa de infiltración & Infil. / Recr. & & & $x$ & $x$ & $x$ & $\mathrm{x}$ & $x$ & $x$ & $x$ & $x$ \\
\hline Canal de infiltración E (Caz Viejo) & Infil. / Trans. & $x$ & $x$ & $x$ & $x$ & $x$ & $\mathrm{x}$ & $x$ & $x$ & $x$ & $x$ \\
\hline Canal Infiltración Nuevo (Ext. E) & Infiltración & & & & & & $x$ & $x$ & $x$ & $x$ & $x$ \\
\hline Canal de infiltración O & Infiltración & & & & $x$ & $x$ & $\mathrm{x}$ & $\mathrm{x}$ & $x$ & $x$ & $x$ \\
\hline EDAR & Depuración & & & & & $x$ & $x$ & $\mathrm{x}$ & $x$ & $x$ & $x$ \\
\hline Canal con biofiltro (tramo C. este) & Dep. / Tran. / Infil. & & & & & $x$ & $x$ & $x$ & $x$ & $\mathrm{x}$ & $x$ \\
\hline Humedales Artificiales 1 y 2 & Dep. / Infil. / Amb. & & & & $x$ & $x$ & $x$ & $\mathrm{x}$ & $\mathrm{x}$ & $\mathrm{x}$ & $x$ \\
\hline Derivación a Laguna alcalina & Transporte & & & & $x$ & $x$ & & & $x$ & $x$ & \\
\hline Restauración Laguna alcalina & Ambiental & & & & $x$ & $x$ & & & $x$ & $x$ & \\
\hline
\end{tabular}




\subsection{Usos del sistema de Recarga Gestionada de Acuíferos de Santiuste}

\subsubsection{Recarga y distribución para riego (balsa, tuberías y canales)}

El sistema pionero del acuífero de Santiuste abastece a unas 2.100 hectáreas de regadío, una superficie creciente por el efecto de garantía de dotación de la recarga. Los volúmenes anuales recargados entre 2002 y 2012 variaron entre 0,46 y $12,19 \mathrm{hm}^{3}$ (Figura 4 a). Desde 2005 se complementaron con otro medio $\mathrm{hm}^{3}$ anual de la EDAR que opera durante todo el año. En la toma del río las derivaciones oscilaron entre 0,52 a $12,68 \mathrm{hm}^{3}$. En 2012 la situación de sequía hizo imposible la recarga activa. La tasa de recarga en todo el sistema oscila entre 37,1 y $96,1 \%$ (Figura 4 b). Si originalmente se estimaban unas 850 ha regadas en la zona, hoy en día se ha consolidado este uso en 2.500 ha servidas por pozos.

Las balsas de decantación e infiltración están operativas desde 2004-05 y ocupan una superficie de $14.340 \mathrm{~m}^{2}$ con una tasa de infiltración $3.000 \mathrm{~mm} / \mathrm{ha}$. En el vaso de la mayor de ellas se han realizado varias experiencias de labrado a distintas distancias de crestas entre surcos de labrado para facilitar la infiltración.

De los dos canales de infiltración se calcula que unos $22,7 \mathrm{~km}$ son de infiltración efectiva (tasa media de $2.500 \mathrm{~mm} / \mathrm{ha}$ ), mientras que unos $3 \mathrm{~km}$ no lo son según los ensayos realizados en la campaña 2004-05. De este modo, en esos tramos el transporte supera a la infiltración por lo que el sistema de 53 dispositivos de parada instalados y las 5 interconexiones permitirían fomentar diferencialmente el traslado frente a la infiltración de volúmenes a distintas sub-cubetas o sectores Norte o Sur en función de las necesidades de riego en cada una.
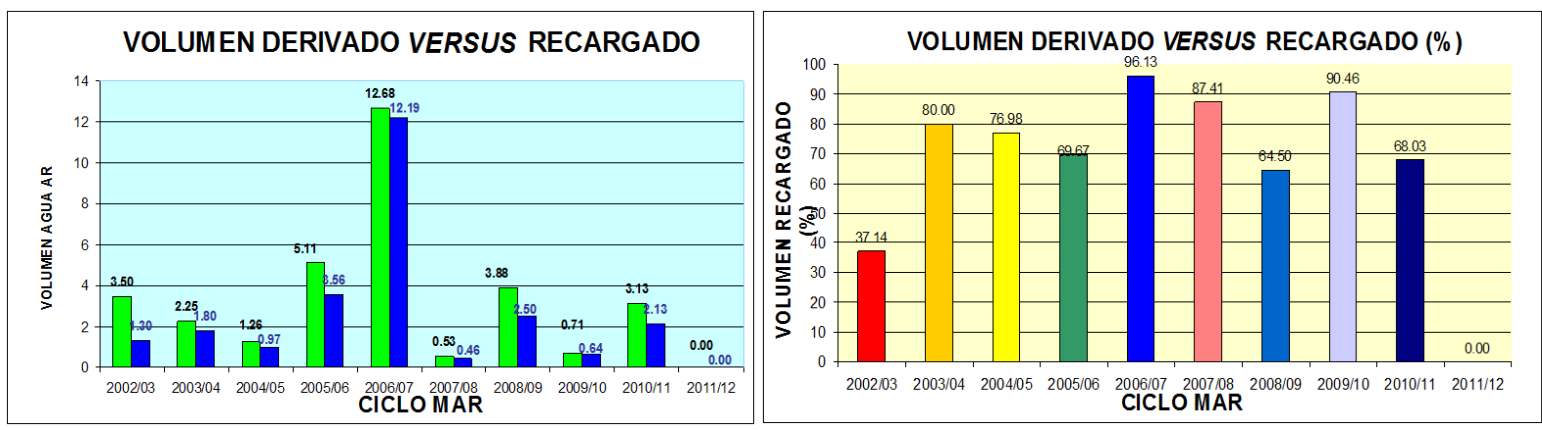

Figura 4 a y b. Gráficos de recarga en $\mathrm{hm}^{3}$ y \% de 2002 a 2012 (Determinación por 54 muestras y método WTF (F. Escalante, 2014)

\subsubsection{Laminación de avenidas (aliviaderos del Sanchón y del Castrillo)}

Los 2 aliviaderos sirven para evitar inundaciones en el entorno regable cuando los canales están repletos y suceden precipitaciones extremas al mismo tiempo. Sin embargo, el dispositivo podría aprovecharse también para la laminación de avenidas extraordinarias en casos de emergencia en el Voltoya mediante la derivación de caudales hasta 1000 $\mathrm{m}^{3} /$ segundo por un circuito de $25 \mathrm{~km}$ de longitud.

\subsubsection{Depuración de aguas (biofiltro en canal)}

La EDAR vierte el agua tratada en 4 balsas de lagunaje en el "caz" Este, que conserva su vegetación natural en este tramo, trabajando como un biofiltro, hasta llegar a un aliviadero, para casos de inundaciones o precipitaciones extremas que devuelve el excedente al Voltoya. El primer tramo es filtrante y ocupa $1.129 \mathrm{~m}$ mientras que el no filtrante se extiende durante $1.577 \mathrm{~m}$ hasta la desembocadura del Humedal $2 \mathrm{~b}$ de retorno al Caz Viejo. El calado y el desarrollo vegetal juegan un papel crucial en los procesos depurativos del agua de mezcla de EDAR y agua del Voltoya. 
Los principales parámetros de calidad del agua sufren una recuperación que se basa en la simple dilución tras el aporte de la EDAR al canal. Sin embargo, en la Tabla 2 se pueden observar 7 parámetros de los analizados en Figura 5 cuyos valores sufren una disminución significativa en el canal ( $\mathrm{Cu}$, Turbidez y Carbono Orgánico Disuelto) y los que la sufren en los Humedales Artificiales (Fe, P, Fosfatos y Amonio). Los parámetros con diferencias inferiores al $12 \%$ se han considerado poco demostrativos.
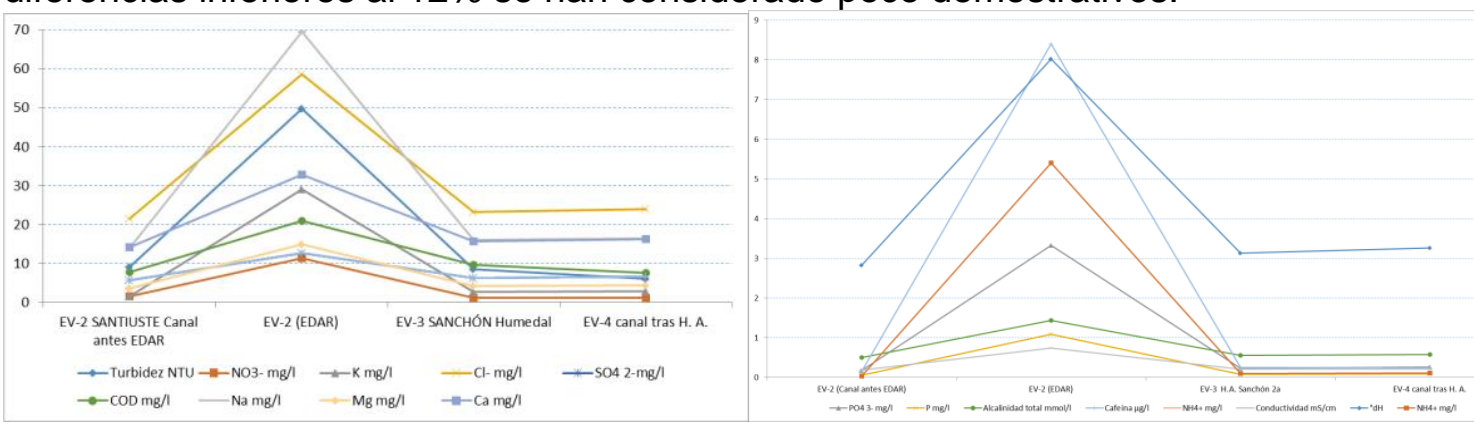

Figura 5 a y b. Gráficos de parámetros de calidad de agua en canal antes y después de la EDAR y de la propia EDAR (17/2/2015)

Tabla 2. Comparativa de parámetros con diferencias significativas a favor del canal (negativos) y a favor del humedal artificial (positivos) 17/2/2015

\begin{tabular}{|c|c|c|c|c|c|c|c|}
\hline Estación & Cu mg/l & $\begin{array}{c}\text { Turbidez } \\
\text { NTU }\end{array}$ & COD mg/l & Fe mg/l & $P$ mg/l & PO4 ${ }^{3-} \mathrm{mg} / \mathrm{l}$ & $\mathrm{NH}^{+} \mathrm{mg} / \mathrm{l}$ \\
\hline EV-2 (Canal antes EDAR) & $100 \%$ & $100 \%$ & $100 \%$ & $100 \%$ & $100 \%$ & $100 \%$ & $100 \%$ \\
\hline EDAR efluente & $74 \%$ & $546 \%$ & $273 \%$ & $63 \%$ & $1902 \%$ & $1910 \%$ & $14211 \%$ \\
\hline EV-3 H. A. Sanchón 2a & $84 \%$ & $94 \%$ & $125 \%$ & $92 \%$ & $130 \%$ & $130 \%$ & $263 \%$ \\
\hline EV-4 Canal tras H. A. & $32 \%$ & $66 \%$ & $97 \%$ & $104 \%$ & $149 \%$ & $149 \%$ & $289 \%$ \\
\hline Diferencia EV4-EV3 & $-53 \%$ & $-28 \%$ & $-27 \%$ & $12 \%$ & $19 \%$ & $20 \%$ & $26 \%$ \\
\hline
\end{tabular}

\subsubsection{Depuración, filtración y restauración ambiental (Humedales Artificiales):}

La presencia de un nivel freático superficial permitió la construcción de dos humedales artificiales tras el canal biofiltrante EN 2005-2006, cuya evolución biológica ayuda a finalizar el proceso de depuración del agua de la EDAR y su infiltración paulatina. Importante refugio faunístico en estío. La vegetación hidrofílica del canal y humedales está compuesta principalmente por Phragmites australis (carrizo) y Scirpus holoschoenus (junco), con otras especies, como Juncus sp. y Typha domingensis (enea). En humedales de baja salinidad se han instalado varias especies hidrofílicas, como la lenteja de agua (Lemna sp.) y del grupo de las algas verdes.

Tabla 3. Análisis de agua en Santiuste. Parámetros inestables in situ (Multiparameter HANNA HI 9829) 17/2/2015

\begin{tabular}{|l|r|r|r|r|r|r|r|r|}
\hline \multicolumn{1}{|c|}{ Estación } & pH & POR [mV] & OD [ppm] & $\begin{array}{c}\text { CE } \\
\text { [uS/cm] }\end{array}$ & $\begin{array}{c}\text { STD } \\
\text { [ppm] }\end{array}$ & $\begin{array}{c}\text { Salinidad } \\
\text { [PSU] }\end{array}$ & $\mathbf{U N T}^{\mathbf{U}}$ & $\begin{array}{c}\mathbf{T}^{\mathbf{a}} \\
{\left[{ }^{\circ} \mathbf{C}\right]}\end{array}$ \\
\hline EV-2 (Canal antes EDAR) & 7 & 247 & 3,62 & 197 & 99 & 0,11 & 8,2 & 6,11 \\
\hline EDAR efluente & 7 & 60 & 0,65 & 720 & 359 & 0,4 & 57,4 & 7,12 \\
\hline EV-2b & 8 & 103,2 & 4,72 & 207 & 104 & 0,11 & 11,1 & 6,36 \\
\hline EV-3 H. A. Sanchón 2a & 8 & 180 & 4,2 & 237 & 118 & 0,13 & 8,4 & 6,8 \\
\hline
\end{tabular}


Los datos de la Tabla 3 muestran como en el humedal (EV-3) respecto al canal previo (EV-2b) el Potencial Redox casi se dobla, pese al descenso del oxígeno disuelto, aumenta la CE y los sólidos disueltos pero baja la turbidez y aumenta la tª․ Los fenómenos de sedimentación y crecimiento bacteriológico o vegetal en la columna pueden justificar estos fenómenos pese contar aún solo con resultados preliminares.

En la Tabla 2 se mostraban los parámetros que se reducen en los humedales artificiales (Fe, P, Fosfatos y Amonio) en relación con el comportamiento del canal, donde el caudal circulante y su morfología no facilitan la formación de un cierto calado y el estancamiento del agua que favorezcan la eliminación diferencial de estos compuestos.

\subsubsection{Restauración ambiental (Laguna alcalina de la Iglesia):}

La laguna de la Iglesia es un humedal alcalino rehabilitado por medio de una solución específica ( $F$. Escalante, 2006). La recuperación de la mineralización, notoria en pH y CE, se logra mediante la interacción entre el flujo superficial del agua de recarga y los sedimentos salinos de la cuenca ribereña (Tabla 4), lo que permite la pervivencia de una colonia de bacterias endémicas (Sanz-Montero et al., 2013), vegetación protegida (Escudero et al., 2008) y un importante refugio de avifauna (Tabla 5).

Tabla 4. Análisis de agua en Santiuste. Parámetros inestables in situ (Multiparameter HANNA HI 9829) 22/1/2009 y 17/2/2015

\begin{tabular}{|l|r|r|r|r|r|r|r|r|r|}
\hline \multicolumn{1}{|c|}{ Estación } & \multicolumn{1}{c|}{$\mathbf{p H}$} & \multicolumn{1}{c|}{$\begin{array}{c}\text { ORP } \\
\text { [mV] }\end{array}$} & $\begin{array}{c}\text { TOD } \\
\text { [ppm] }\end{array}$ & $\begin{array}{c}\text { CE } \\
\text { [uS/cm] }\end{array}$ & $\begin{array}{c}\text { STD } \\
\text { [ppm] }\end{array}$ & $\begin{array}{c}\text { Salinidad } \\
\text { [PSU] }\end{array}$ & UNT & $\begin{array}{c}\text { Ta } \\
{\left[{ }^{\circ} \mathbf{C}\right]}\end{array}$ & $\begin{array}{c}\mathbf{P} \\
{[\mathbf{m b a r}]}\end{array}$ \\
\hline $\begin{array}{l}\text { Tubería Salida } \\
\text { 22/1/09 }\end{array}$ & 6,33 & 22,4 & 10,2 & 253 & - & 0,13 & - & 7,2 & \\
\hline $\begin{array}{l}\text { Laguna de la Iglesia } \\
\text { 22/1/09 }\end{array}$ & - & $-25,8$ & 4,9 & 2.184 & - & 1,13 & - & 9,32 & \\
\hline $\begin{array}{l}\text { Tubería Salida } \\
\text { 17/2/15 }\end{array}$ & 7 & 121,7 & 14,5 & 244 & 123 & 0,14 & 8,2 & 5,88 & 935 \\
\hline $\begin{array}{l}\text { Laguna de la Iglesia } \\
\text { 17/2/15 }\end{array}$ & 9 & 115 & 5,77 & 5.397 & 2.703 & 3,37 & 570 & 8,01 & 937,7 \\
\hline
\end{tabular}

En caso de humedales con procesos hidroquímicos alcalinizantes y reductores en sus aguas predominan procesos de desnitrificación y carbonatación, con el subsiguiente incremento de la alcalinidad, que favorece la descomposición de la materia orgánica y el desarrollo de organismos en el fango (Desir et al., 2003).

Tabla 5. Inventario de especies en la Laguna de la Iglesia (2007/2012//2015)

\begin{tabular}{|c|c|c|c|c|c|c|c|c|c|}
\hline $\begin{array}{l}\text { NOMBRE } \\
\text { COMÚN }\end{array}$ & $\begin{array}{l}\text { NOMBRE } \\
\text { CIENTíFICO }\end{array}$ & ธิे & 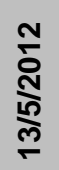 & 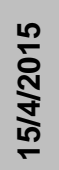 & $\begin{array}{l}\text { NOMBRE } \\
\text { COMÚN }\end{array}$ & $\begin{array}{c}\text { NOMBRE } \\
\text { CIENTíFICO }\end{array}$ & ڤิे & $\begin{array}{l}\stackrel{N}{5} \\
\stackrel{N}{N} \\
\stackrel{N}{\sigma}\end{array}$ & $\frac{10}{5}$ \\
\hline $\begin{array}{l}\text { Aguilucho } \\
\text { cenizo }\end{array}$ & Circus pygargus & & & $\mathrm{X}$ & Garza real & Ardea cinerea & $\mathrm{X}$ & & \\
\hline Ánade real & $\begin{array}{l}\text { Anas } \\
\text { platyrhynchos }\end{array}$ & $\mathrm{X}$ & $\mathrm{X}$ & $\mathrm{X}$ & $\begin{array}{l}\text { Golondrina } \\
\text { común }\end{array}$ & Hirudo rustica & & & $\mathrm{X}$ \\
\hline Ánade friso & Anas strepera & & $\mathrm{X}$ & $\mathrm{X}$ & L. cascadeña & Motacilla cinerea & & & $\mathrm{X}$ \\
\hline Ánade rabudo & Anas acuta & $x$ & & & Milano negro & Milvus migrans & & $\mathrm{X}$ & $\mathrm{X}$ \\
\hline Ánade silbón & Anas penelope & $\mathrm{X}$ & & & Pato colorado & Netta rufina & $\mathrm{X}$ & & \\
\hline $\begin{array}{l}\text { Andarríos } \\
\text { chico }\end{array}$ & Actitis hypoleucos & & $X$ & & Pato cuchara & Anas clypeata & $\mathrm{X}$ & $x$ & $\mathrm{X}$ \\
\hline Avefría & Vanellus vanellus & $\mathrm{X}$ & & $\mathrm{X}$ & Polla de agua & Gallinula chloropus & $\mathrm{X}$ & & \\
\hline Avoceta & $\begin{array}{l}\text { Recurvirostra } \\
\text { avosetta }\end{array}$ & & $\mathrm{X}$ & & Tarro blanco & Tadorna tadorna & $\mathrm{X}$ & & $x$ \\
\hline Cerceta & Anas querquedula & & & $\mathrm{X}$ & Tórtola común & Streptopelia turtur & & $\mathrm{X}$ & \\
\hline
\end{tabular}




\begin{tabular}{|l|l|c|c|c|l|l|l|l|l|}
\hline carretona & & & & & & & & \\
\hline Cerceta común & Anas creca & $\mathrm{X}$ & & & Triguero & Miliaria calandra & $\mathrm{X}$ & $\mathrm{X}$ \\
\hline Chorlitejo & Charadrius spp. & & $\mathrm{X}$ & & $\begin{array}{l}\text { Zampullín } \\
\text { chico }\end{array}$ & Tachybaptus ruficollis & $\mathrm{X}$ & $\mathrm{X}$ \\
\hline Cigüeñuela & $\begin{array}{l}\text { Himantopus } \\
\text { himantopus }\end{array}$ & $\mathrm{X}$ & $\mathrm{X}$ & $\mathrm{X}$ & $\begin{array}{l}\text { Zampullín } \\
\text { cuellinegro }\end{array}$ & Podiceps negricollis & $\mathrm{X}$ & \\
\hline Focha común & Fulica atra & $\mathrm{X}$ & & $\mathrm{X}$ & & & & & \\
\hline
\end{tabular}

La primera franja perilagunar corresponde con el transepto de especies subhalófitas y alcalinófitas, asociadas a zonas de descarga de sistemas de flujos intermedios (Rey Benayas, 1991). Según este autor, las especies inventariadas e indicadoras de esta franja del perfil son las siguientes (sic): Alopecurus geniculatus, Deschampsia cespitosa subsp. refracta, Rumex conglomerata, Festuca arundinacea subsp. fenas, Juncus acutus, Juncus gerarsi, Scirpus maritimus subsp. compactus

La siguiente franja perilagunar en los humedales salinos está ocupada por especies halófitas asociadas a aguas subterráneas muy mineralizadas y envejecidas, como son: Aerulopus littoralis, Artemisia caerulescens, Convolvulus lineatus, Frankenia lavéis, Juncus maritimus, Juncus subulanus, Limonium costae y Suaeda vera.

La zona periférica de protección u orla perilagunar de carácter salino de los suelos con ocasional erosión del horizonte A se conoce como salguero, y presenta eflorescencias bien en un cinturón o bien desconectadas en manchas blancas y grises de sales en el sustrato. En conjunto constituyen los "prados salinos." En ocasiones hay especies adaptadas a la salinidad, como los tarays (Tamarix canariensis) y otras especies más comunes en lugares salinos, como Suaeda splendens, Salicornia europea, Chenopodium cf. quenopodialis y Kickxia cf. Spuria.

\section{CONCLUSIONES Y EXPECTATIVAS}

Los dispositivos implementados cumplen simultáneamente diversos papeles:

- Recarga subterránea de fuente superficial en temporada de aguas altas. 10 años de recarga contrastada por datos de derivación, tasa de recarga, longitud de canal filtrante, superficies de infiltración, inversión, días de funcionamiento...

- Distribución de recursos por una zona regable alimentada por pozos. Las tuberías, los canales interconectados y la infiltrabilidad diferencial permiten trasladar caudales de sur a norte sin coste energético por $15 \mathrm{~km}$ de zona regable.

- Ahorro en bombeo por subida de piezometría. Según los datos proporcionados por la CR de Santiuste, para bombeo medio para riego de $9950 \mathrm{~m}^{3} /$ pozo, y a 0,106986 $€ / \mathrm{kWh}$; el ahorro anual en consumo directo de energía por pozo se estima en 27,1 $\mathrm{kWh} / \mathrm{m}$ lo que equivale a $2,90 € / \mathrm{m}$. Es decir, por cada metro de nivel freático ganado, se ahorran $27,1 \mathrm{kWh}(2,90 €$ a la tarifa dada).

- Mejora de calidad del agua: Disminución de $\mathrm{NO}_{3}(29 \%)$, $\mathrm{Cu}(68 \%)$ y turbidez (34\%) en canal (EV-4) respecto al agua de entrada del Voltoya (EV-2).

- Biodepuración pasiva por dilución, sedimentación y bioactividad por todos los parámetros de canal (EV-4) respecto a EDAR y por los datos de Fe, P, Fosfatos y Amonio en humedales (EV-3) respecto al propio canal

- Gestión de vertidos. El sistema de recarga absorbe el efluente de la EDAR (incluso fuera de campaña) haciéndolo circular por los biofiltros de canales y humedales. 
- Laminación de avenidas: Aliviaderos de Sanchón y Castrillejo hacia los ríos Voltoya y Eresma respectivamente con capacidad extra en el humedal Sanchón 2b.

- Fomento de biodiversidad: Uso contrastado de hasta 25 especies de aves en la laguna de la Iglesia y en menor medida de los humedales artificiales y canales.

- Protección de endemismos: Especies de alto valor de la Laguna de la Iglesia con la Comunidad Arctodiaptomion con Arctodiaptomus salinus y Cletocamptus retrogressus (Crustacea), además de Cyanobacterias, diatomeas, ostrácodos (Crustacea)...

- Creación de elementos socio-culturales: Las láminas de agua de balsas, lagunas y humedales han generado en su entorno áreas de interés paisajístico y recreativo.

Sobre todos las demás funciones, la recarga cuenta con el importante incentivo del apoyo y cooperación de la sociedad rural que se beneficia del sistema como lo demuestra la colaboración en la gestión mantenimiento y participación de ayuntamientos y regantes en las labores de recarga, tareas relacionadas con el seguimiento e $\mathrm{I}+\mathrm{D}+\mathrm{i}$ y sesiones de difusión del conocimiento entre los usuarios y habitantes.

Estos datos son el punto de partida para la generación de indicadores que sirvan para valorar el funcionamiento de los sistemas de recarga y la comparación entre dispositivos bajo condiciones técnicas y funcionales equivalentes dentro del marco del proyecto europeo MARSOL (Schüth et al., 2014).

\section{AGRADECIMIENTOS}

Este artículo ha sido redactado por miembros del equipo del Grupo Tragsa del proyecto europeo MARSOL (Demostrating Managed Aquifer Recharge as a solution to water scarcity and drought) (WP5), con el apoyo de la European Commission, convocatoria Water Inno-demo (FP 7) con Grant Agreement: GA 619120. El artículo refleja el punto de vista de los autores y la Comisión no tiene responsabilidad sobre las opiniones publicadas en el mismo.

\section{BIBLIOGRAFÍA}

Desir, G., Gtez. Elorza, M. \& Gtez. Santolalla, F. (2003). Origen y evolución de las playas en una zona semiárida con arenas eólicas (región de Coca, Cuenca del Duero, España). Boletín Geológico y Minero 114(4), pp. 395-407.

Escudero, A. et al. (2008). Guía básica para la interpretación de los hábitats de interés comunitario en Castilla y León. Junta de Castilla y León. Consejería de Medio Ambiente. Valladolid. $432 \mathrm{pp}$.

Fdez. Escalante, E. (2006). Los humedales. Detección de impactos ambientales y posibilidades de regeneración hídrica mediante la gestión de la recarga artificial. El ejemplo de la laguna de la Iglesia, Villagonzalo de Coca (Segovia). Tecnologí@ y Desarrollo. Revista de ciencia, tecnología y medio ambiente. Volumen IV. Separata.

Fdez. Escalante, E. (2014). 2002-2012, Una década de recarga gestionada. Acuífero de la Cubeta de Santiuste (Castilla y León). Libro, Serie hidrogeología Hoy título 7, Ed. Tragsa, Madrid ISBN: 84-616-8910-0, 298 pp. 
Galán López, R., Fdez. Escalante, E. \& Mtez. Rubio, J. (2001). Contribuciones al estudio hidrogeológico para la recarga artificial del acuífero de la Cubeta de Santiuste. (Segovia). VII Simposio de hidrogeología, AEH, Murcia.

Rey Benayas, J. M. (1991) Aguas subterráneas y ecología. Ecosistemas de descarga de acuíferos en los arenales. Colección Técnica 139, ICONA, Madrid 141 pp.

Sanz Montero, M. E. et al. (2013). Procesos de sedimentación y biomineralización en la laguna alcalina de las Eras (Humedal Coca-Olmedo). Geogaceta 53. ISSN 0213-683X.

Schüth, C. et al. (2014) MARSOL Demonstrating Managed Aquifer Recharge as a Solution to Water Scarcity and Drought. EGU General Assembly 2014, Geophysical Research Abstracts, Vol. 16, EGU2014-9073. 